\title{
The Marshall-Olkin Half Logistic-G Family of Distributions With Applications
}

\author{
Boikanyo Makubate $^{1}$, Fastel Chipepa ${ }^{1,2}$, Broderick Oluyede ${ }^{1} \&$ Peter O. Peter $^{1}$ \\ ${ }^{1}$ Department of Mathematics and Statistical Sciences, Botswana International University of Science and Technology, P. \\ Bag 16, Palapye, Botswana \\ 2 Department of Applied Mathematics and Statistics, Midlands State University, P. Bag 9055, Gweru, Zimbabwe \\ Correspondence: Department of Mathematics and Statistical Sciences, Botswana International University of Science and \\ Technology, P. Bag 16, Palapye, Botswana. E-mail: chipepaf@msu.ac.zw
}

Received: October 20, 2020 Accepted: February 21, 2021 Online Published: February 24, 2021

doi:10.5539/ijsp.v10n2p120 URL: https://doi.org/10.5539/ijsp.v10n2p120

\begin{abstract}
Attempts have been made to define new classes of distributions that provide more flexibility for modeling data that is skewed in nature. In this work, we propose a new family of distributions namely the Marshall-Olkin Half Logistic-G (MOHL-G) based on the generator pioneered by [Marshall and Olkin , 1997]. This new family of distributions allows for a flexible fit to real data from several fields, such as engineering, hydrology, and survival analysis. The structural properties of these distributions are studied and its model parameters are obtained through the maximum likelihood method. We finally demonstrate the effectiveness of these models via simulation experiments.
\end{abstract}

Keywords: Marshall-Olkin-G, Half-Logistic-G, maximum likelihood estimation

\section{Introduction}

More work has recently been done by various authors on the development of new families of distributions through the extension of other existing continuous distributions. These new families have found wider applicability in modeling data in different areas such as engineering, economics, biological studies, environmental sciences, just to mention these few. The main objectives of generalizing this new family of distributions are as follows: achieve skewness for symmetrical models; develop special models exhibiting various shapes of hazard rate function; develop heavy-tailed distributions useful in many real data sets; attain more flexible kurtosis compared to that of the baseline distribution; generate skewed, symmetric, J-shaped or reversed-J shaped distributions with better fits than other generalized distributions having the same underlying model.

[Marshall and Olkin , 1997], proposed a new distribution with cumulative distribution function (cdf) and probability density function (pdf) given by

and

$$
F_{M O-G}(x ; \delta, \xi)=1-\frac{\delta \bar{G}(x ; \xi)}{1-\bar{\delta} \bar{G}(x ; \xi)}
$$

$$
f_{M O-G}(x ; \delta, \xi)=\frac{\delta g(x ; \xi)}{[1-\bar{\delta} \bar{G}(x ; \xi)]^{2}},
$$

respectively, where $\delta$ denotes the tilt parameter and $G(x ; \xi)$ is the baseline cdf. The distribution is more flexible compared to the exponential, Weibull and gamma distributions.

Furthermore, [Cordeiro et al. , 2016], developed the type 1 half-logistic family of distributions with the cdf and pdf given by

$$
\begin{aligned}
F(x ; \lambda, \xi) & =\int_{0}^{-\ln (1-G(x ; \xi))} \frac{2 \lambda \exp \{-\lambda x\}}{(1+\exp \{-\lambda x\})^{2}} d x \\
& =\frac{1-[1-G(x ; \xi)]^{\lambda}}{1+[1-G(x ; \xi)]^{\lambda}},
\end{aligned}
$$

where $G(x ; \xi)$ is the cdf of the baseline distribution and $\lambda>0$ is the shape parameter. We obtain a special case, namely, half-logistic-G (HL-G) model, with cdf

$$
F_{H L-G}(x ; \xi)=\frac{G(x ; \xi)}{1+\bar{G}(x ; \xi)},
$$


if we set $\lambda=1$ in equation (3). The corresponding pdf of the HL-G model is given by

$$
f_{H L-G}(x ; \xi)=\frac{2 g(x ; \xi)}{(1+\bar{G}(x ; \xi))^{2}} .
$$

The Marshall-Olkin distribution has been generalized from several distributions to construct many family of distributions achieving some of the above characteristics. The examples of these include; Marshall-Olkin-G (MO-G) family by [Marshall and Olkin , 1997], beta Marshall-Olkin-G (BMO-G) by [Alizadeh et al. , 2015a], Kumaraswamy Marshall-Olkin-G (KwMO-G) by [Alizadeh et al. , 2015b], among others. The proposed distribution gives a new flexible parametric model useful in modeling various forms of data in reliability studies, survival analysis, statistical mechanics, quality control, economics, bio medical studies, etc. [Lepetu et al. , 2017], introduced a new class of distributions called the Marshall-Olkin Log-logistic Extended Weibull (MOLLEW) family of distributions. Their work employs the Marshall-Olkin transformation to the Log-logistic Weibull distribution to obtain more new flexible models suitable for reliability data.

Marshall- Olkin transformation was applied to various well-known distributions: Weibull by [Ghitany et al. , 2005], [Zhang and Xie , 2007]. Lately, the general results have been addressed by [Barreto-Souza et al. , 2013]. [Santos-Neo et al. , 2014], introduces a new class of models called the Marshall-Olkin extended Weibull family of distributions which defines at least twenty-one special models. [Chakraborty and Handique , 2017], presented the generalized Marshall-Olkin Kumaraswamy-G distribution. [Lazhar , 2017] developed and studied the properties of the Marshall-Olkin extended generalized Gompertz distribution. [Kumar, 2016] discussed the ratio and inverse moments of Marshall-Olkin extened Burr Type III distribution from lower generalized order statistics.

On the similar note, the Half-Logistic (HL) distribution has not received much attention from researchers in terms of generalization. [Afify et al. , 2017] proposed a new flexible family of distributions called the Odd exponentiated half logistic-G (OEHL-G) family of distributions using the HL distribution as the generator and studied its mathematical properties. Their results utilized the flexibility of the baseline distribution in modeling various forms of data.

Other generally known families are the beta-G by [Eugene et al. , 2002], odd log-logistic-G by [Gleaton and Lynch , 2004], the transmuted-G by [Shaw and Buckley , 2009], the gamma-G by [Zografos and Balakrishnan , 2009], the Kumaraswamy-G by [Cordeiro and de Castro , 2011], the logistic-G by [Torabi and Montazeri , 2014], exponentiated generalized-G by [Cordeiro et al. , 2013], the McDonald-G by [Alexander et al. , 2012], T-X family by [Alzaatreh et al. , 2013], the exponentiated half-logistic generated family by [Cordeiro et al. , 2014], the beta odd log-logistic generalized by [Cordeiro et al. , 2015], generalized transmuted-G by [Nofal et al. , 2017], generalized odd log-logistic-G by [Cordeiro et al. , 2017]. Various structural properties of the extended distributions may be easily explored using mixture forms of exponentiated-G (exp-G) distributions. It is in the same vein that we develop the new family of distributions called Marshall-Olkin Half Logistic-G (MO-HL-G) family of distributions.

The layout of this paper is as follows; Section 2, presents the new generalized family of distributions with some of its structural properties. The Maximum likelihood estimates are derived under Section 3. Some of the special cases of this new generalized distributions are presented in Section 4. Section 5, presents results from Monte Carlo simulation study. In Section 6. we present applications of the proposed model to real data sets and finally give concluding remarks under Section 7.

\section{The Model and Statistical Properties}

\subsection{The Model}

We develop the MO-HL-G distribution using the generalization proposed by [Marshall and Olkin , 1997], and taking the baseline distribution to be the HL-G distribution by [Cordeiro et al. , 2016]. The cdf and pdf of the MO-HL-G family of distributions is given by

$$
F_{M O-H L-G}(x ; \delta, \xi)=\frac{\left[\frac{G(x ; \xi)}{1+\bar{G}(x ; \xi)}\right]}{1-\bar{\delta}\left[1-\frac{G(x ; \xi)}{1+\bar{G}(x ; \xi)}\right]}
$$

and

$$
f_{M O-H L-G}(x ; \delta, \xi)=\frac{2 \delta g(x ; \xi)}{[1+\bar{G}(x ; \xi)]^{2}\left[1-\bar{\delta}\left(1-\frac{G(x ; \xi)}{1+\bar{G}(x ; \xi)}\right)\right]^{2}},
$$

respectively, for $\delta>0, \bar{\delta}=1-\delta$ and $\xi$ is a vector of parameters from the baseline distribution function $G($.$) .$

\subsection{Quantile Function}

The quantile function for the MO-HL-G family of distributions is derived by solving the non-linear equation 


$$
F_{M O-H L-G}(x ; \delta, \xi)=\frac{\left[\frac{G(x ; \xi)}{1+\bar{G}(x ; \xi)}\right]}{1-\bar{\delta}\left[1-\frac{G(x ; \xi)}{1+\bar{G}(x ; \xi)}\right]}=u
$$

for $0 \leq u \leq 1$, that is,

$$
\begin{aligned}
\left(\frac{G(x ; \xi)}{1+\bar{G}(x ; \xi)}\right) & =\left(\frac{u(1-\bar{\delta})}{1-u \bar{\delta}}\right) \\
G(x ; \xi) & =2\left(\left(\frac{u(1-\bar{\delta})}{1-u \bar{\delta}}\right)^{-1}+1\right)^{-1} .
\end{aligned}
$$

Therefore, the quantiles of the MO-HL-G family of distributions may be obtained by solving the equation

$$
x(u)=G^{-1}\left[2\left(\left(\frac{u(1-\bar{\delta})}{1-u \bar{\delta}}\right)^{-1}+1\right)^{-1}\right]
$$

via iterative methods in $\mathrm{R}$ or Matlab software.

\subsection{Expansion of Density}

This sub-section presents statistical properties of the MO-HL-G family of distributions as derived from the general results for the Marshall and Olkin's family of distributions by [Barreto-Souza et al. , 2013]. Considering

$$
f_{M O-H L-G}(x ; \delta, \xi)=\frac{\alpha f_{H L-G}(x ; \xi)}{\left(1-\bar{\delta} \bar{F}_{H L-G}(x ; \xi)\right)^{2}},
$$

we can write equation (7) as

$$
f_{M O-H L-G}(x ; \delta, \xi)=\frac{f_{H L-G}(x ; \xi)}{\delta\left[1-\frac{\delta-1}{\delta} F_{H L-G}(x ; \xi)\right]^{2}},
$$

where $f_{H L-G}(x ; \xi)$ and $F_{H L-G}(x ; \xi)$ are as given in equations (5) and (4), respectively. We apply the series expansion

$$
(1-z)^{-k}=\sum_{j=0}^{\infty} \frac{\Gamma(k+j)}{\Gamma(k) j !} z^{j},
$$

which is valid for $|z|<1$ and $k>0$. If $\delta \in(0,1)$, we can obtain

$$
f_{M O-H L-G}(x ; \delta, \xi)=f_{H L-G}(x ; \xi) \sum_{j=0}^{\infty} \sum_{k=0}^{j} w_{j, k} F_{H L-G}(x ; \xi)^{j-k},
$$

where $w_{j, k}=w_{j, k}(\delta)=\delta(j+1)(1-\delta)^{j}(-1)^{j-k}\left(\begin{array}{l}j \\ k\end{array}\right)$. For $\delta>1$, we have

$$
f_{M O-H L-G}(x ; \delta, \xi)=f_{H L-G}(x ; \xi) \sum_{j=0}^{\infty} v_{j} F_{H L-G}^{j}(x ; \xi),
$$

where $v_{j}=v_{j}(\delta)=\frac{(j+1)(1-1 / \delta)}{\delta}$. For $\delta \in(0,1)$, equation (7) becomes

$$
\begin{aligned}
f_{\text {MO-HL-G }}(x ; \delta, \xi) & =\frac{2 g(x ; \xi)}{(1+\bar{G}(x ; \xi))^{2}} \sum_{j=0}^{\infty} \sum_{k=0}^{j} w_{j, k}\left[\frac{G(x ; \xi)}{1+\bar{G}(x ; \xi)}\right]^{j-k} \\
& =\sum_{j=0}^{\infty} \sum_{k=0}^{j} 2 w_{j, k} g(x, \xi) \frac{[G(x ; \xi)]^{j-k}}{[1+\bar{G}(x ; \xi)]^{j-k+2}} .
\end{aligned}
$$

Using the series expansion

$$
[1+\bar{G}(x ; \xi)]^{-(j-k+2)}=\sum_{m=0}^{\infty}(-1)^{m}\left(\begin{array}{c}
-(j-k+2) \\
m
\end{array}\right) \bar{G}^{m}(x ; \xi)
$$

and

$$
\bar{G}^{m}(x ; \xi)=[1-G(x ; \xi)]^{m}=\sum_{l=0}^{\infty}(-1)^{l}\left(\begin{array}{c}
m \\
l
\end{array}\right) G^{l}(x ; \xi),
$$


we can write

$$
\begin{aligned}
f_{M O-H L-G}(x ; \delta, \xi) & =\sum_{j, m, l=0}^{\infty} \sum_{k=0}^{j} \frac{2(-1)^{m+l}}{l+j-k+1} w_{j, k}\left(\begin{array}{c}
-(j-k+2) \\
m
\end{array}\right)\left(\begin{array}{c}
m \\
l
\end{array}\right) \\
& \times(l+j-k+1) g(x ; \xi)[G(x ; \xi)]^{l+j-k} \\
& =\sum_{j, m, l=0}^{\infty} w_{j, m, l}^{*} g_{l+j-k}(x ; \xi) .
\end{aligned}
$$

It follows that for $\delta \in(0,1)$, the MO-HL-G family of distributions can be expressed as an infinite linear combination of the exponentiated-G (Exp-G) distribution with power parameter $(l+j-k)$ and linear component

$$
w_{j, m, l}^{*}=\sum_{k=0}^{j} \frac{2(-1)^{m+l}}{l+j-k+1} w_{j, k}\left(\begin{array}{c}
-(j-k+2) \\
m
\end{array}\right)\left(\begin{array}{c}
m \\
l
\end{array}\right) .
$$

Furthermore, for $\delta>1$ equation (7) can be written as

$$
\begin{aligned}
f_{M O-H L-G}(x ; \delta, \xi) & =\frac{2 g(x ; \xi)}{(1+\bar{G}(x ; \xi))^{2}} \sum_{j=0}^{\infty} v_{j}\left[\frac{G(x ; \xi)}{1+\bar{G}(x ; \xi)}\right]^{j} \\
& =\sum_{j=0}^{\infty} 2 v_{j} \frac{g(x ; \xi)[G(x ; \xi)]^{j}}{[1+\bar{G}(x ; \xi)]^{j+2}} .
\end{aligned}
$$

Applying the series expansion

$$
[1+\bar{G}(x ; \xi)]^{-(j+2)}=\sum_{m=0}^{\infty}(-1)^{m}\left(\begin{array}{c}
-(j+2) \\
m
\end{array}\right) \bar{G}^{m}(x ; \xi)
$$

and

$$
\bar{G}^{m}(x ; \xi)=[1-G(x ; \xi)]^{m}=\sum_{l=0}^{\infty}(-1)^{l}\left(\begin{array}{c}
m \\
l
\end{array}\right) G^{l}(x ; \xi),
$$

we can write

$$
\begin{aligned}
f_{M O-H L-G}(x ; \delta, \xi) & =\sum_{j, m, l=0}^{\infty} \frac{2(-1)^{m+l}}{l+j+1} v_{j}\left(\begin{array}{c}
-(j+2) \\
m
\end{array}\right)\left(\begin{array}{c}
m \\
l
\end{array}\right) \\
& \times(l+j+1) g(x ; \xi)[G(x ; \xi)]^{l+j} \\
& =\sum_{j, m, l=0}^{\infty} v_{j, m, l}^{*} g_{l+j}(x ; \xi) .
\end{aligned}
$$

Therefore, for $\delta>1$, the MO-HL-G family of distributions can be expressed as a linear combination of the Exp-G distribution with power parameter $(l+j)$ and linear component

$$
v_{j, m, l}^{*}=\frac{2(-1)^{m+l}}{l+j+1} v_{j}\left(\begin{array}{c}
-(j+2) \\
m
\end{array}\right)\left(\begin{array}{c}
m \\
l
\end{array}\right) .
$$

\subsection{Distribution of Order Statistics}

Suppose that $X_{1}, X_{2}, \ldots, X_{n}$ are independent and identically distributed (i.i.d) random variables distributed according to (7). The pdf of the $i^{\text {th }}$ order statistic $X_{i: n}$, is given by

$$
f_{i: n}(x ; \delta, \xi)=\delta n ! f_{H L-G}(x ; \xi) \sum_{l=0}^{n-i} \frac{(-1)^{l}}{(i-1) !(n-i) !} \frac{F_{H L-G}^{l+i-1}(x ; \xi)}{\left[1-\bar{\delta} F_{H L-G}(x ; \xi)\right]^{l+i-1}} .
$$

If $\delta \in(0,1)$, we have

$$
f_{i: n}(x ; \delta, \xi)=f_{H L-G}(x ; \xi) \sum_{j=0}^{\infty} \sum_{l=0}^{n-i} \sum_{k=0}^{j} U_{j, l, k} F_{H L-G}^{j+l-k+i-1}(x ; \xi)
$$


where

$$
U_{j, l, k}=U_{j, l, k}(\delta)=\frac{\delta n !(-1)^{l}(1-\delta)^{j}(-1)^{j-k}}{(i-1) !(n-i) !}\left(\begin{array}{l}
j \\
k
\end{array}\right)\left(\begin{array}{c}
l+i+j \\
j
\end{array}\right) .
$$

For $\delta>1$, we write $1-\bar{\delta} \bar{F}_{H L-G}(x ; \xi)=\delta\left\{1-(\delta-1) F_{H L-G}(x ; \xi) / \delta\right\}$, such that

$$
f_{i: n}(x ; \delta, \xi)=f_{H L-G}(x ; \xi) \sum_{j=0}^{\infty} \sum_{l=0}^{n-i} c_{j, l} F_{H L-G}^{j+l+i-1}(x ; \xi),
$$

where

$$
c_{j, l}=c_{j, l}(\delta)=\frac{(-1)^{l}(\delta-1)^{j} n !}{\delta^{l+j+i}(i-1) !(n-i) !}\left(\begin{array}{c}
l+i+j \\
j
\end{array}\right) .
$$

For $\delta \in(0,1)$, using equation (19) and substituting $f(x)$ by equation (5) and $F(x)$ by equation (4), we get

$$
\begin{aligned}
f_{i: n}(x ; \delta, \xi) & =\frac{2 g(x ; \xi)}{(1+\bar{G}(x ; \xi))^{2}} \sum_{j=0}^{\infty} \sum_{l=0}^{n-i} \sum_{k=0}^{j} U_{j, l, k}\left[\frac{G(x ; \xi)}{1+\bar{G}(x ; \xi)}\right]^{j+l-k+i-1} \\
& =\sum_{j=0}^{\infty} \sum_{l=0}^{n-i} \sum_{k=0}^{j} 2 U_{j, l, k} \frac{g(x ; \xi)[G(x ; \xi)]^{j+l-k+i-1}}{[1+\bar{G}(x ; \xi)]^{j+l-k+i+1}} .
\end{aligned}
$$

By applying the expansions

$$
[1+\bar{G}(x ; \xi)]^{-(j+l-k+i+1)}=\sum_{m=o}^{\infty}(-1)^{m}\left(\begin{array}{c}
-(j+l-k+i+1) \\
m
\end{array}\right) \bar{G}^{m}(x ; \xi)
$$

and

$$
\bar{G}^{m}(x ; \xi)=[1-G(x ; \xi)]^{m}=\sum_{p=0}^{\infty}(-1)^{p}\left(\begin{array}{c}
m \\
p
\end{array}\right) G^{p}(x ; \xi),
$$

we can write

$$
\begin{aligned}
f_{i: n}(x ; \delta, \xi) & =\sum_{j, m, p=0}^{\infty} \sum_{l=0}^{n-i} \sum_{k=0}^{j} \frac{2(-1)^{m+p}}{p+j+l-k+i} U_{j, l, k}\left(\begin{array}{c}
-(j+l-k+i+1) \\
m
\end{array}\right)\left(\begin{array}{l}
m \\
p
\end{array}\right) \\
& \times(p+j+l-k+i) g(x ; \xi)[G(x ; \xi)]^{p+j+l-k+i-1} \\
& =\sum_{j, m, p=0}^{\infty} U_{j, m, p}^{*} g_{p+j+l-k+i}(x ; \xi),
\end{aligned}
$$

where $g_{p+j+l-k+i}(x ; \xi)=(p+j+l-k+i) g(x ; \xi)[G(x ; \xi)]^{p+j+l-k+i-1}$ is an Exp-G distribution with power parameter $(p+j+l-k+i)$ and

$$
U_{j, m, p}^{*}=\sum_{l=0}^{n-i} \sum_{k=0}^{j} \frac{2(-1)^{m+p}}{p+j+l-k+i} U_{j, l, k}\left(\begin{array}{c}
-(j+l-k+i+1) \\
m
\end{array}\right)\left(\begin{array}{l}
m \\
p
\end{array}\right) .
$$

Furthermore, for $\delta>1$, we get

$$
\begin{aligned}
f_{i: n}(x ; \delta, \xi) & =\frac{2 g(x ; \xi)}{(1+\bar{G}(x ; \xi))^{2}} \sum_{j=0}^{\infty} \sum_{i=0}^{n-i} c_{j, l}\left[\frac{G(x ; \xi)}{1+\bar{G}(x ; \xi)}\right]^{j+l+i-1} \\
& =\sum_{j=0}^{\infty} \sum_{i=0}^{n-i} 2 c_{j, l} \frac{g(x ; \xi)[G(x ; \xi)]^{j+l+i-1}}{[1+\bar{G}(x ; \xi)]^{j+l+i+1}}
\end{aligned}
$$

By applying the binomial expansions

$$
[1+\bar{G}(x ; \xi)]^{-(j+l+i+1)}=\sum_{m=o}^{\infty}(-1)^{m}\left(\begin{array}{c}
-(j+l+i+1) \\
m
\end{array}\right) \bar{G}^{m}(x ; \xi)
$$


and

$$
\bar{G}^{m}(x ; \xi)=[1-G(x ; \xi)]^{m}=\sum_{p=0}^{\infty}(-1)^{p}\left(\begin{array}{c}
m \\
p
\end{array}\right) G^{p}(x ; \xi),
$$

we can write

$$
\begin{aligned}
f_{i: n}(x ; \delta, \xi) & =\sum_{j, m, p=0}^{\infty} \sum_{i=0}^{n-i} \frac{2(-1)^{m+p}}{p+j+l+i} c_{j, l}\left(\begin{array}{c}
-(j+l+i+1) \\
m
\end{array}\right)\left(\begin{array}{c}
m \\
p
\end{array}\right) \\
& \times(p+j+l+i) g(x ; \xi)[G(x ; \xi)]^{p+j+l+i-1} \\
& =\sum_{j, m, p=0}^{\infty} c_{j, m, p}^{*} g_{p+j+l+i}(x ; \xi),
\end{aligned}
$$

where $g_{p+j+l+i}(x ; \xi)=(p+j+l+i) g(x ; \xi)[G(x ; \xi)]^{p+j+l+i-1}$ is an Exp-G distribution with power parameter $(p+j+l+i)$ and

$$
c_{j, m, p}^{*}=\sum_{i=0}^{n-i} \frac{2(-1)^{m+p}}{p+j+l+i} c_{j, l}\left(\begin{array}{c}
-(j+l+i+1) \\
m
\end{array}\right)\left(\begin{array}{l}
m \\
p
\end{array}\right) .
$$

\subsection{Entropy}

An Entropy gives a measure of variation of uncertainty for a random variable $X$ with pdf $g(x)$. There are two common measures of entropy, namely Shannon entropy by [Shannon, 1951] and Rényi entropy by [Rényi , 1961]. Rényi entropy is defined by

$$
I_{R}(v)=(1-v)^{-1} \log \left[\int_{0}^{\infty} g^{v}(x) d x\right]
$$

where $v>0$ and $v \neq 1$. Using expansion (11), for $\delta \in(0,1)$

$$
f_{M O-H L-G}^{v}(x ; \delta, \xi)=\frac{\delta^{v} f_{H L-G}^{v}(x ; \xi)}{\Gamma(2 v)} \sum_{j=0}^{\infty}(1-\delta)^{j} \Gamma(2 v+j) \frac{\left[1-F_{H L-G}(x ; \xi)\right]^{j}}{j !}
$$

and for $\delta>1$

$$
f_{M O-H L-G}^{v}(x ; \delta, \xi)=\frac{f_{H L-G}^{v}(x)}{\delta^{\nu} \Gamma(2 v)} \sum_{j=0}^{\infty}(\delta-1)^{j} \Gamma(2 v+j) \frac{F_{H L-G}^{j}(x ; \xi)}{j !} .
$$

Thus, Rényi entropy for $\delta \in(0,1)$ and $\delta>1$ are given by

$$
I_{R}(v)=(1-v)^{-1} \log \left(\sum_{j=0}^{\infty} e_{j} \int_{0}^{\infty} f_{H L-G}^{v}(x ; \xi)\left(1-F_{H L-G}(x ; \xi)\right)^{j} d x\right)
$$

and

$$
I_{R}(v)=(1-v)^{-1} \log \left(\sum_{j=0}^{\infty} h_{j} \int_{0}^{\infty} f_{H L-G}^{v}(x ; \xi) F_{H L-G}^{j}(x ; \xi) d x\right)
$$

where

$$
e_{j}=e_{j}(\delta)=\frac{\delta^{v}(1-\delta)^{j} \Gamma(2 v+j)}{\Gamma(2 v) j !}
$$

and

$$
h_{j}=h_{j}(\delta)=\frac{(\delta-1)^{j} \Gamma(2 v+j)}{\delta^{v+j} \Gamma(2 v) j !} .
$$

Now, for $\delta \in(0,1)$ and using equation (27), we have

$$
I_{R}(v)=(1-v)^{-1} \log \left[\sum_{j=0}^{\infty} e_{j} \int_{0}^{\infty} \frac{2^{v} g^{v}(x ; \xi)}{(1+\bar{G}(x ; \xi))^{2 v}}\left[1-\frac{G(x ; \xi)}{1+\bar{G}(x ; \xi)}\right]^{j} d x\right] .
$$

By considering the following expansions

$$
\left[1-\frac{G(x ; \xi)}{1+\bar{G}(x ; \xi)}\right]^{j}=\sum_{m=0}^{\infty}(-1)^{m} \frac{\Gamma(j+1)}{\Gamma(j+1-m) m !}\left[\frac{G(x ; \xi)}{1+\bar{G}(x ; \xi)}\right]^{m},
$$




$$
(1+\bar{G}(x ; \xi))^{-(2 v+m)}=\sum_{p=0}^{\infty}(-1)^{p}\left(\begin{array}{c}
-(2 v+m) \\
p
\end{array}\right) \bar{G}^{p}(x ; \xi)
$$

and

$$
[\bar{G}(x ; \xi)]^{p}=[1-G(x ; \xi)]^{p}=\sum_{q=0}^{\infty}(-1)^{q}\left(\begin{array}{l}
p \\
q
\end{array}\right) G^{q}(x ; \xi),
$$

we can write

$$
\begin{aligned}
I_{R}(v) & =(1-v)^{-1} \log \left[\sum_{j, m, p, q=0}^{\infty} e_{j} \frac{2^{v}(-1)^{p+m+q} \Gamma(j+1)}{\Gamma(j+1-m) m !}\left(\begin{array}{c}
-(2 v+m) \\
p
\end{array}\right)\left(\begin{array}{l}
p \\
q
\end{array}\right)\right. \\
& \left.\times \frac{1}{\left(\frac{m+q}{v}+1\right)^{v}} \int_{0}^{\infty}\left(\left(\frac{m+q}{v}+1\right) g(x ; \xi)[G(x ; \xi)]^{\frac{m+q}{v}}\right)^{v} d x\right] \\
& =(1-v)^{-1} \log \left[\sum_{j, m, p, q=0}^{\infty} e_{j, m, p, q}^{*} \exp (1-v) I_{R E G}\right],
\end{aligned}
$$

where

$$
e_{j, m, p, q}^{*}=e_{j} \frac{2^{v}(-1)^{p+m+q} \Gamma(j+1)}{\Gamma(j+1-m) m !}\left(\begin{array}{c}
-2 v \\
p
\end{array}\right)\left(\begin{array}{c}
p-m \\
q
\end{array}\right) \frac{1}{\left(\frac{m+q}{v}+1\right)^{v}}
$$

and $I_{R E G}=\int_{0}^{\infty}\left(\left(\frac{m+q}{v}+1\right) g(x ; \xi)[G(x ; \xi)]^{\frac{m+q}{v}}\right)^{v} d x$ is the Rényi entropy of the Exp-G distribution with power parameter $\frac{m+q}{v}$. Furthermore, for $\delta>1$

$$
I_{R}(v)=(1-v)^{-1} \log \left[\sum_{j=0}^{\infty} h_{j} \int_{0}^{\infty} \frac{2^{v} g^{v}(x ; \xi)}{(1+\bar{G}(x ; \xi))^{2 v}}\left[\frac{G(x ; \xi)}{1+\bar{G}(x ; \xi)}\right]^{j} d x\right] .
$$

By considering the following expansions

$$
(1+\bar{G}(x ; \xi))^{-(2 v+j)}=\sum_{m=0}^{\infty}(-1)^{m}\left(\begin{array}{c}
-(2 v+j) \\
m
\end{array}\right) \bar{G}^{m}(x ; \xi)
$$

and

$$
[\bar{G}(x ; \xi)]^{m}=[1-G(x ; \xi)]^{m}=\sum_{p=0}^{\infty}(-1)^{p}\left(\begin{array}{c}
m \\
p
\end{array}\right) G^{p}(x ; \xi),
$$

we can write

$$
\begin{aligned}
I_{R}(v) & =(1-v)^{-1} \log \left[\sum_{j, m, p=0}^{\infty} h_{j} 2^{v}(-1)^{p+m}\left(\begin{array}{c}
-(2 v+j) \\
m
\end{array}\right)\left(\begin{array}{c}
m \\
p
\end{array}\right) \frac{1}{\left(\frac{p+j}{v}+1\right)^{v}}\right. \\
& \left.\times \int_{0}^{\infty}\left(\left(\frac{p+j}{v}+1\right) g(x ; \xi)[G(x ; \xi)]^{\frac{p+j}{v}}\right)^{v} d x\right] \\
& =(1-v)^{-1} \log \left[\sum_{j, m, p=0}^{\infty} h_{j, m, p}^{*} \exp (1-v) I_{R E G}\right],
\end{aligned}
$$

where

$$
h_{j, m, p}^{*}=h_{j} 2^{v}(-1)^{p+m}\left(\begin{array}{c}
-(2 v+j) \\
m
\end{array}\right)\left(\begin{array}{c}
m \\
p
\end{array}\right) \frac{1}{\left(\frac{p+j}{v}+1\right)^{v}}
$$

and $I_{R E G}=\int_{0}^{\infty}\left(\left(\frac{p+j}{v}+1\right) g(x ; \xi)[G(x ; \xi)]^{\frac{p+j}{v}}\right)^{v} d x$ is the Rényi entropy of the Exp-G distribution with power parameter $\frac{p+j}{v}$. 


\subsection{Moments and Generating Functions}

Let $X \sim M O-H L-G(\delta, \xi)$, then the $r^{\text {th }}$ moment can be obtained from equations (14) and (16). For $\delta \in(0,1)$,

$$
E\left(X^{r}\right)=\sum_{j, m, l=0}^{\infty} w_{j, m, l}^{*} E\left(W_{l+j-k}^{r}\right),
$$

where $w_{j, m, l}^{*}$ is as defined in equation (15) and $E\left(W_{l+j-k}^{r}\right)$ denotes the $r^{\text {th }}$ moment of $W_{l+j-k}$ which follows an Exp-G distribution with power parameter $(l+j-k)$. For $\delta>1$

$$
E\left(X^{r}\right)=\sum_{j, m, l=0}^{\infty} v_{j, m, l}^{*} E\left(W_{l+j}^{r}\right),
$$

where $v_{j, m, l}^{*}$ is as defined in equation (17) and $E\left(W_{l+j}^{r}\right)$ denotes the $r^{\text {th }}$ moment of $W_{l+j}$ which follows an Exp-G distribution with power parameter $(l+j)$. The incomplete moments can be obtained as follows:

For $\delta \in(0,1)$

$$
I_{X}(t)=\int_{0}^{t} x^{s} f_{M O-H L-G}(x ; \xi) d x=\sum_{j, m, l=0}^{\infty} w_{j, m, l}^{*} I_{l+j-k}(t),
$$

where $I_{l+j-k}(t)=\int_{0}^{t} x^{r} g_{l+j-k}(x ; \xi) d x$ and $w_{j, m, l}^{*}$ is as defined in equation (15). Also, For $\delta>1$

$$
I_{X}(t)=\int_{0}^{t} x^{s} f_{M O-H L-G}(x ; \xi) d x=\sum_{j, m, l=0}^{\infty} v_{j, m, l}^{*} I_{l+j}(t),
$$

where $I_{l+j}(t)=\int_{0}^{t} x^{r} g_{l+j}(x ; \xi) d x$ and $v_{j, m, l}^{*}$ is as defined in equation (17). The moment generating function (mgf) of $X$ is as follows:

For $\delta \in(0,1)$

$$
M_{X}(t)=\sum_{j, m, l=0}^{\infty} w_{j, m, l}^{*} E\left(e^{t W_{l+j-k}}\right),
$$

where $E\left(e^{t W_{l+j-k}}\right)$ is the mgf of the Exp-G distribution with power parameter $(l+j-k)$ and $w_{j, m, l}^{*}$ is as defined in equation (15). For $\delta>1$

$$
M_{X}(t)=\sum_{j, m, l=0}^{\infty} v_{j, m, l}^{*} E\left(e^{t W_{l+j}}\right),
$$

where $E\left(e^{t W_{l+j}}\right)$ is the mgf of the Exp-G distribution with power parameter $(l+j)$ and $v_{j, m, l}^{*}$ is as defined in equation (17). Furthermore, we can get the characteristic function and is given by $\phi(t)=E\left(e^{i t X}\right)$, where $i=\sqrt{-1}$, for $\delta \in(0,1)$

$$
\phi(t)=\sum_{j, m, l=0}^{\infty} w_{j, m, l}^{*} \phi_{l+j-k}(t),
$$

where $\phi_{l+j-k}(t)$ is the characteristic function of Exp-G distribution with power parameter $(l+j-k)$ and $w_{j, m, l}^{*}$ is as defined in equation (15). For $\delta>1$

$$
\phi(t)=\sum_{j, m, l=0}^{\infty} v_{j, m, l}^{*} \phi_{l+j}(t),
$$

where $\phi_{l+j}(t)$ is the characteristic function of Exp-G distribution with power parameter $(l+j)$ and $v_{j, m, l}^{*}$ is as defined in equation (17).

\section{Maximum Likelihood Estimation}

If $X_{i} \sim M O-H L-W(\delta ; \xi)$ with the parameter vector $\Delta=(\delta ; \xi)^{T}$. The total $\log$-likelihood $\ell=\ell(\Delta)$ from a random sample of size $n$ is given by

$$
\ell=n \log (2 \delta)+\sum_{i=1}^{n} \log \left[g\left(x_{i} ; \xi\right)\right]-2 \sum_{i=1}^{n} \log \left[1+\bar{G}\left(x_{i} ; \xi\right)\right] 2 \sum_{i=1}^{n} \log \left[1-\bar{\delta}\left(1-\frac{G\left(x_{i} ; \xi\right)}{1+\bar{G}\left(x_{i} ; \xi\right)}\right)\right] .
$$


The elements of a score vector, $U=\left(\frac{\partial \ell}{\partial \delta}, \frac{\partial \ell}{\partial \xi_{k}}\right)$ are given by:

$$
\frac{\partial \ell}{\partial \delta}=\frac{n}{\delta}-2 \sum_{i=1}^{n} \frac{1-\frac{G\left(x_{i} ; \xi\right)}{1+\bar{G}\left(x_{i} ; \xi\right)}}{\left[1-\bar{\delta}\left(1-\frac{G\left(x_{i} ; \xi\right)}{1+\bar{G}\left(x_{i} ; \xi\right)}\right)\right]}
$$

and

$$
\begin{aligned}
\frac{\partial \ell}{\partial \xi_{k}} & =\sum_{i=1}^{n} \frac{1}{g\left(x_{i} ; \xi\right)} \frac{\partial g\left(x_{i} ; \xi\right)}{\partial \xi_{k}}-2 \sum_{i=1}^{n} \frac{1}{\left[1+\bar{G}\left(x_{i} ; \xi\right)\right]} \frac{\partial\left[1+\bar{G}\left(x_{i} ; \xi\right)\right]}{\partial \xi_{k}} \\
& -2 \sum_{i=1}^{n} \frac{1}{\left[1-\bar{\delta}\left(1-\frac{G\left(x_{i} ; \xi\right)}{1+\bar{G}\left(x_{i} ; \xi\right)}\right)\right]} \frac{\partial\left[1-\bar{\delta}\left(1-\frac{G\left(x_{i} ; \xi\right)}{1+\bar{G}\left(x_{i} ; \xi\right)}\right)\right]}{\partial \xi_{k}}
\end{aligned}
$$

respectively. These partial derivatives does not have closed form and can be solved using numerical methods such as Newton-Raphson procedure..

\section{Some Special Cases}

In this Section, we present some special cases of the MO-HL-G family of distributions. We considered cases when the baseline distributions are log-logistic, Weibull and normal distributions.

\subsection{Marshall-Olkin-Half Logistic-Log-Logistic Distribution}

Consider the log-logistic distribution as the baseline distribution. The log-logistic distribution has pdf and cdf given by $g(x)=c x^{c-1}\left(1+x^{c}\right)^{-2}$ and $G(x)=1-\left(1+x^{c}\right)^{-1}$, for $c>0$, respectively. Therefore, the Marshall-Olkin-Half LogisticLog-Logistic (MO-HL-LLo) distribution have the cdf and pdf given by

$$
F_{M O-H L-L L o}(x ; \delta, c)=\frac{1-\left(1+x^{c}\right)^{-1}}{\left[1+\left(1+x^{c}\right)^{-1}\right]\left[1-\bar{\delta}\left(1-\frac{1-\left(1+x^{c}\right)^{-1}}{1+\left(1+x^{c}\right)^{-1}}\right)\right]}
$$

and

$$
f_{M O-H L-L L o}(x ; \delta, c)=\frac{2 \delta c x^{c-1}\left(1+x^{c}\right)^{-2}}{\left[1+\left(1+x^{c}\right)^{-1}\right]^{2}\left[1-\bar{\delta}\left(1-\frac{1-\left(1+x^{c}\right)^{-1}}{1+\left(1+x^{c}\right)^{-1}}\right)\right]^{2}},
$$

respectively, for $\delta, c>0$.
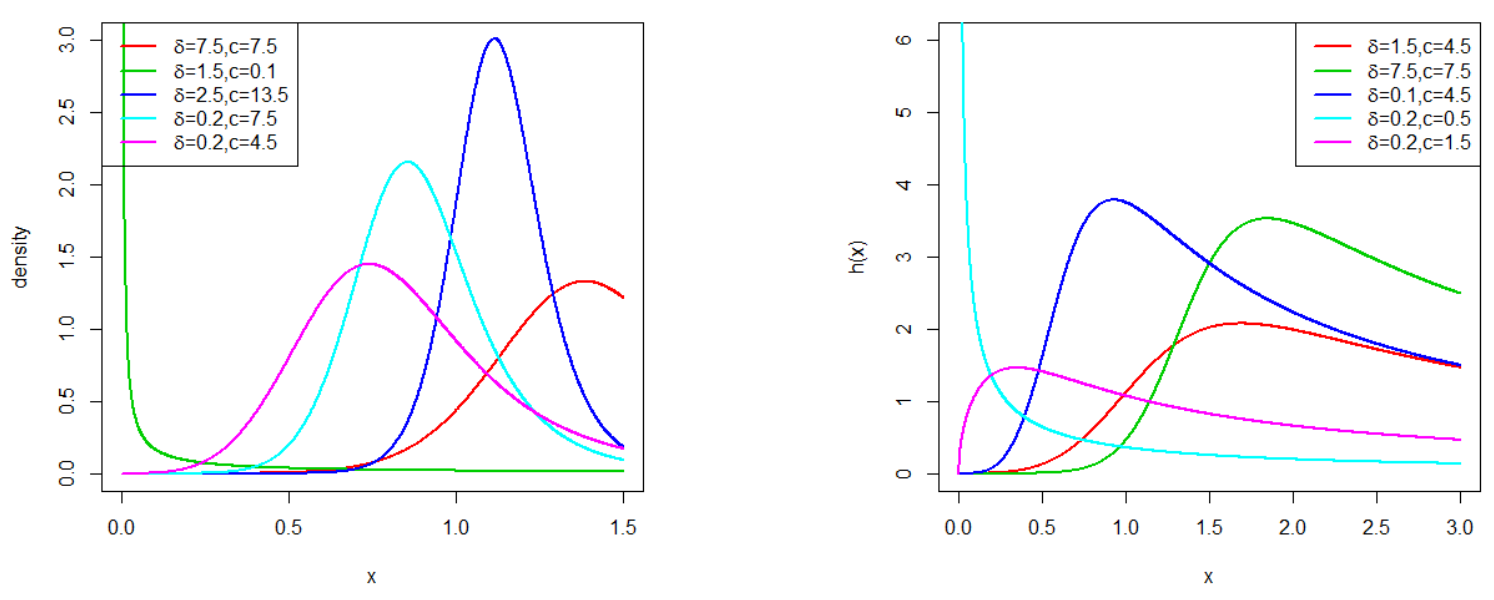

Figure 1. Plots of the pdf and hrf for the MO-HL-LLo distribution

Figure 1 shows the plots of the pdfs and hrfs of the MO-HL-LLo distribution for selected parameters values. The pdf can take various shapes including reverse-J, unimodal, left and right skewed. The MO-HL-LLo distribution exhibits both monotonic and non-monotonic hazard rate functions. 


\subsection{Marshall-Olkin Half Logistic-Weibull Distribution}

Consider the Weibull distribution as the baseline distribution with pdf and cdf given by $g(x ; \lambda, \omega)=\lambda \omega x^{\omega-1} e^{-\lambda x^{\omega}}$ and $G(x ; \lambda, \omega)=1-e^{-\lambda x^{\omega}}$, respectively, for $\lambda, \omega>0$. The cdf and pdf of the Marshall-Olkin-half-logistic-Weibull (MO-HLW) distribution are given by

$$
F_{M O-H L-W}(x ; \delta, \lambda, \gamma)=\frac{1-e^{\lambda x^{\omega}}}{\left(1+e^{\lambda x^{\omega}}\right)\left[1-\bar{\delta}\left(1-\frac{1-e^{\lambda \chi^{\ell \omega}}}{1+e^{\lambda x^{\omega}}}\right)\right]}
$$

and

$$
f_{M O-H L-W}(x ; \delta, \lambda, \gamma)=\frac{2 \delta \lambda \omega x^{\omega-1} e^{-\lambda x^{\omega}}}{\left(1+e^{\lambda x^{\alpha \omega}}\right)^{2}\left[1-\bar{\delta}\left(1-\frac{1-e^{\lambda \lambda^{\omega \omega}}}{1+e^{\lambda \lambda^{\omega}}}\right)\right]^{2}},
$$

respectively, for $\delta, \lambda, \omega>0$.
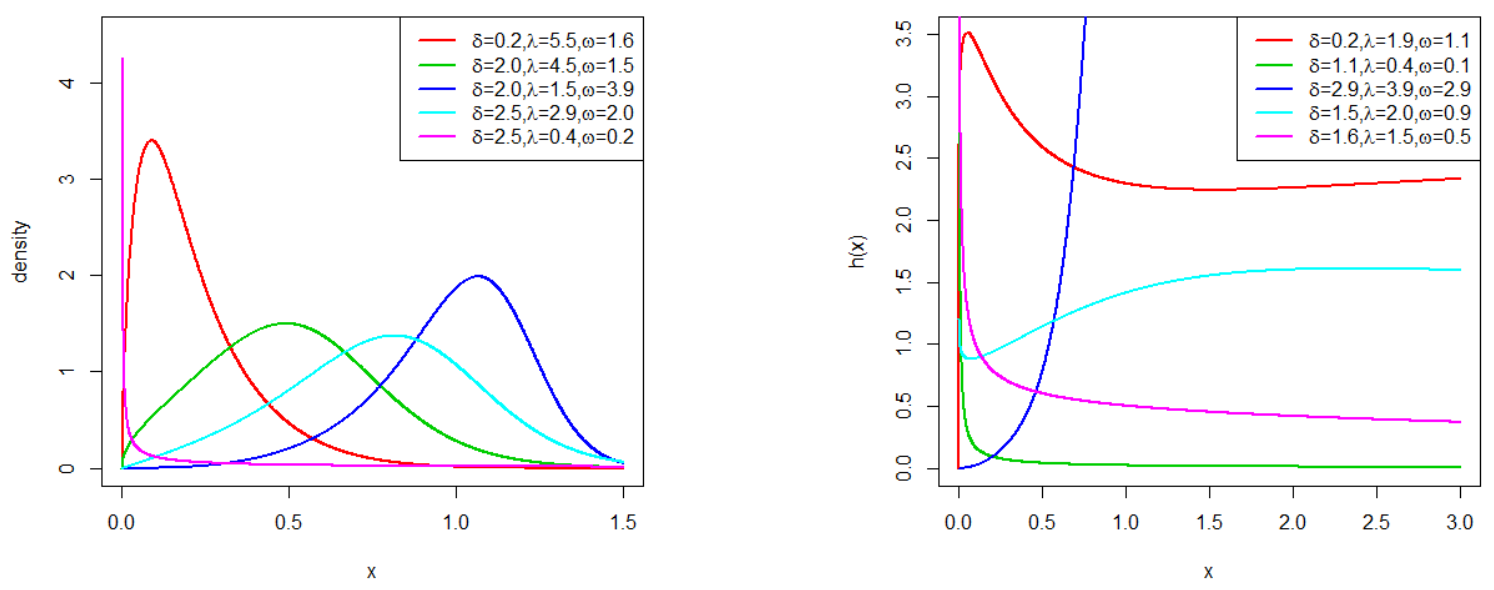

Figure 2. Plots of the pdf and hrf for the MO-HL-W distribution

The pdfs shown in Figure 2 of the MO-HL-W distribution can take various shapes that include reverse-J, uni-modal, left or right skewed shapes. Furthermore, the MO-HL-W distribution exhibit increasing, decreasing, reverse-J, bathtub and upside bathtub shapes for the hazard rate function.

\subsection{Marshall-Olkin-Half Logistic-Normal Distribution}

Consider the normal distribution with pdf $g(x ; \mu, \sigma)=\sigma^{-1} \phi\left(\frac{x-\mu}{\sigma}\right)$ and $\operatorname{cdf} G(x ; \mu, \sigma)=\Phi\left(\frac{x-\mu}{\sigma}\right)$, for $\mu \in \mathfrak{R}$ and $\sigma>0$, as the baseline distribution, we obtain the Marshall-Olkin-half-logistic-normal (MO-HL-N) distribution with cdf and pdf given by

$$
F_{M O-H L-N}(x ; \delta, \mu, \sigma)=\frac{\Phi\left(\frac{x-\mu}{\sigma}\right)}{\left(2-\Phi\left(\frac{x-\mu}{\sigma}\right)\right)\left[1-\bar{\delta}\left(1-\frac{\Phi\left(\frac{x-\mu}{\sigma}\right)}{2-\Phi\left(\frac{x-\mu}{\sigma}\right)}\right)\right]}
$$

and

$$
f_{M O-H L-N}(x ; \delta, \mu, \sigma)=\frac{2 \delta \sigma^{-1} \phi\left(\frac{x-\mu}{\sigma}\right)}{\left(2-\Phi\left(\frac{x-\mu}{\sigma}\right)\right)^{2}\left[1-\bar{\delta}\left(1-\frac{\Phi\left(\frac{x-\mu}{\sigma}\right)}{2-\Phi\left(\frac{x-\mu}{\sigma}\right)}\right)\right]^{2}},
$$

for $\delta, \sigma>0$ and $-\infty<\mu<\infty$.

Figure 3 shows that the MO-HL-N distribution can take various shapes for its pdf. Also, the hazard rate function for the MO-HL-N distribution exhibit different shapes for the hazard rate functions for the MO-HL-N distribution. 

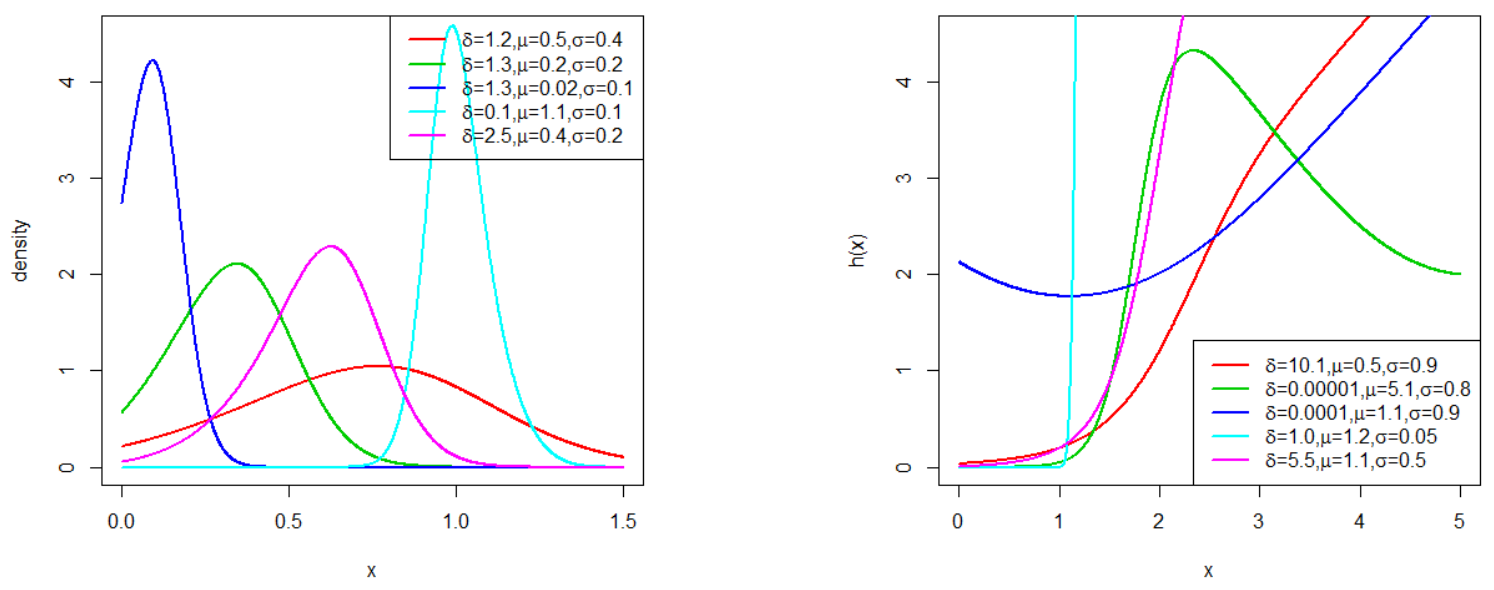

Figure 3. Plots of the pdf and hrf for the MO-HL-N distribution

\section{Simulation Study}

A simulation study is conducted to evaluate consistency of the maximum likelihood estimators. We simulated for $\mathrm{N}=1000$ times with sample size $n=50,100,200,400,800$ and 1000. Simulation results are shown in Table 1. From the Monte Carlo simulation results, we conclude that our model produces consistent results when estimating parameters for the model.

\section{Applications}

In this Section, three real data examples are used to illustrate the usefulness of the proposed new model compared to other competing known non-nested models. We use the following goodness-of-fit statistics: -2loglikelihood (-2 $\log \mathrm{L})$, Akaike Information Criterion (AIC), Consistent Akaike Information Criterion (AICC), Bayesian Information Criterion (BIC), Cramér von Mises $\left(W^{*}\right)$ and Andersen-Darling $\left(A^{*}\right)$ as described by [Chen and Balakrishnan, 1995], KolmogorovSminorv (K-S) and its p-value to assess the best fitting model. The model with the smaller values of these statistics, is deemed to be the better model.

We used the nlm function in R software to estimate the model parameters and the package AdequacyModel in $\mathbf{R}$ software for goodness-of-fit test. Model parameters estimates (standard errors in parenthesis) and the goodness-of-fit-statistics for the three data sets are shown in Tables 2, 3 and 4. Plots of the fitted densities, the histogram of the data and probability plots (Chambers, Cleveland, Kleiner and Tukey (1983)) are also presented to show how well our model fits the observed data sets compared to the selected non-nested models. The plots are shown in Figures 4 (a), 4 (b), 5 (a), 5 (b), 6 (a) and 6 (b).

We compared the MO-HL-W distribution with other competing three parameter non-nested models: the exponentiatedFréchet (EFr) distribution by [Nadarajah and Kotz , 2003], other two non-nested studied by [Barreto-Souza et al. , 2013], namely, Marshall-Olkin extended Fréchet (MOEFr) and Marshall-Olkin extended generalized exponential (MOEGE) distributions, Marshall-Olkin extended inverse Weibull (IWMO) by [Pakungwati et al. , 2018], exponentiated Weibull by [Pal et al. , 2006] and alpha power Weibull (APW) by [Nassar et al. , 2018] distributions. The pdfs of the non-nested models are given by:

$$
f_{I W M O}(x ; \alpha, \theta \lambda)=\frac{\alpha \lambda \theta^{-\lambda} x^{-\lambda-1} e^{-(\theta x)^{-\lambda}}}{\left[\alpha-(\alpha-1) e^{-(\theta x)^{-\lambda}}\right]^{2}},
$$

for $\alpha, \theta, \lambda>0$,

$$
f_{E F}(x ; \alpha, \lambda, \delta)=\alpha \lambda \delta^{\lambda}\left[1-e^{-(\delta / x)^{\lambda}}\right]^{\alpha-1} x^{-(1+\lambda)} e^{-(\lambda+1)(\delta / x)^{\lambda}},
$$

for $\alpha, \lambda, \delta>0$,

for $\alpha, \lambda, \delta>0$,

$$
f_{M O E F}(x ; \alpha, \lambda, \delta)=\frac{\alpha \lambda \delta^{\lambda} x^{-(\lambda+1)} e^{-(\delta / x)^{\lambda}}}{\left[1-\bar{\alpha}\left(1-e^{-(\delta / x)^{\lambda}}\right)\right]^{2}},
$$

$$
f_{M O E G E}(x ; \alpha, \gamma, \lambda)=\frac{\alpha \gamma \lambda e^{-\lambda x}\left(1-e^{-\lambda x}\right)^{\gamma-1}}{\left(1-\bar{\alpha}\left[1-e^{-\lambda x}\right]^{\gamma}\right)^{2}},
$$


Table 1. Monte Carlo Simulation Results for MO-HL-W Distribution: Mean, RMSE and Average Bias

\begin{tabular}{|c|c|c|c|c|c|c|c|}
\hline & & \multicolumn{3}{|c|}{$\delta=0.3, \lambda=0.02, \omega=0.3$} & \multicolumn{3}{|c|}{$\bar{c} \delta=0.04, \lambda=0.04, \omega=0.7$} \\
\hline parameter & $\mathrm{n}$ & Mean & RMSE & Bias & Mean & RMSE & Bias \\
\hline \multirow{6}{*}{$\delta$} & 50 & 1.762498 & 9.685856 & 1.462498 & 0.610298 & 6.655783 & 0.570298 \\
\hline & 100 & 0.844508 & 3.890994 & 0.544508 & 0.129558 & 1.395083 & 0.089558 \\
\hline & 200 & 0.699785 & 4.770718 & 0.399785 & 0.063553 & 0.049943 & 0.023553 \\
\hline & 400 & 0.342086 & 0.147265 & 0.042086 & 0.049088 & 0.023075 & 0.009088 \\
\hline & 800 & 0.320848 & 0.090219 & 0.020848 & 0.046309 & 0.017197 & 0.006309 \\
\hline & 1000 & 0.314583 & 0.069455 & 0.014583 & 0.044346 & 0.013374 & 0.004346 \\
\hline \multirow{6}{*}{$\lambda$} & 50 & 0.079121 & 0.229997 & 0.059121 & 0.130856 & 0.350873 & 0.090856 \\
\hline & 100 & 0.048857 & 0.138366 & 0.028857 & 0.078191 & 0.148263 & 0.038191 \\
\hline & 200 & 0.035782 & 0.126931 & 0.015782 & 0.061161 & 0.045279 & 0.021161 \\
\hline & 400 & 0.023250 & 0.013159 & 0.003249 & 0.048323 & 0.022022 & 0.008323 \\
\hline & 800 & 0.021462 & 0.008276 & 0.001462 & 0.046057 & 0.016502 & 0.006057 \\
\hline & 1000 & 0.020888 & 0.006446 & 0.000888 & 0.044062 & 0.013070 & 0.004062 \\
\hline \multirow{6}{*}{$\omega$} & 50 & 0.280205 & 0.060672 & -0.019795 & 0.672670 & 0.106492 & -0.027330 \\
\hline & 100 & 0.285469 & 0.044548 & -0.014531 & 0.685898 & 0.076765 & -0.014102 \\
\hline & 200 & 0.291384 & 0.033146 & -0.008616 & 0.686340 & 0.055460 & -0.013660 \\
\hline & 400 & 0.296389 & 0.021142 & -0.003611 & 0.694657 & 0.036994 & -0.005343 \\
\hline & 800 & 0.298494 & 0.015789 & -0.001506 & 0.694497 & 0.026790 & -0.005503 \\
\hline & 1000 & 0.298659 & 0.012699 & -0.001341 & 0.697050 & 0.023444 & -0.002950 \\
\hline & & \multicolumn{3}{|c|}{$\delta=0.04, \lambda=0.7, \omega=0.7$} & \multicolumn{3}{|c|}{$\delta=0.05, \lambda=0.05, \omega=0.6$} \\
\hline \multirow{6}{*}{$\delta$} & 50 & 0.081044 & 0.100232 & 0.041044 & 0.406615 & 4.491051 & 0.356615 \\
\hline & 100 & 0.309052 & 5.419012 & 0.269052 & 0.169623 & 1.752614 & 0.119623 \\
\hline & 200 & 0.049754 & 0.034046 & 0.009754 & 0.072667 & 0.053849 & 0.022667 \\
\hline & 400 & 0.040278 & 0.017388 & 0.000278 & 0.059569 & 0.026896 & 0.009569 \\
\hline & 800 & 0.034577 & 0.008367 & -0.005423 & 0.055151 & 0.019439 & 0.005151 \\
\hline & 1000 & 0.035456 & 0.007580 & -0.004544 & 0.053182 & 0.015412 & 0.003182 \\
\hline \multirow{6}{*}{$\lambda$} & 50 & 1.122168 & 0.849914 & 0.422168 & 0.127529 & 0.280013 & 0.077529 \\
\hline & 100 & 0.950882 & 0.645455 & 0.250882 & 0.089910 & 0.175096 & 0.039910 \\
\hline & 200 & 0.813907 & 0.396657 & 0.113907 & 0.069754 & 0.047828 & 0.019754 \\
\hline & 400 & 0.709154 & 0.229924 & 0.009154 & 0.058419 & 0.025297 & 0.008419 \\
\hline & 800 & 0.645285 & 0.098588 & -0.054715 & 0.054782 & 0.018518 & 0.004782 \\
\hline & 1000 & 0.668169 & 0.083547 & -0.031831 & 0.052771 & 0.014996 & 0.002771 \\
\hline \multirow{6}{*}{$\omega$} & 50 & 0.712711 & 0.092397 & 0.012711 & 0.583070 & 0.088722 & -0.016930 \\
\hline & 100 & 0.713895 & 0.078494 & 0.013895 & 0.588429 & 0.066197 & -0.011571 \\
\hline & 200 & 0.714713 & 0.054730 & 0.014713 & 0.590984 & 0.047204 & -0.009016 \\
\hline & 400 & 0.719261 & 0.040985 & 0.019261 & 0.595801 & 0.032625 & -0.004199 \\
\hline & 800 & 0.721728 & 0.036012 & 0.021728 & 0.597247 & 0.023541 & -0.002753 \\
\hline & 1000 & 0.723451 & 0.034163 & 0.023451 & 0.599234 & 0.020848 & -0.000766 \\
\hline
\end{tabular}

for $\alpha, \gamma, \lambda>0$,

$$
f_{E W}(x ; \alpha, \beta, \delta)=\alpha \beta \delta x^{\beta-1} e^{-\alpha x^{\beta}}\left(1-e^{-\alpha x^{\beta}}\right)^{\delta},
$$

for $\alpha, \beta, \delta>0$ and

$$
f_{A P W}(x ; \alpha, \beta, \theta)=\frac{\log (\alpha)}{(\alpha-1)} \beta \theta x^{\beta-1} e^{-\theta x^{\beta}} \alpha^{1-e^{-\theta x^{\beta}}}
$$

for $\alpha, \beta, \theta>0$.

\subsection{Strengths of $1.5 \mathrm{~cm}$ Glass Fibres Data}

The first data set is on strengths of $1.5 \mathrm{~cm}$ glass fibres. The data set was also analyzed by [Bourguignon et al. , 2014] and [Smith and Naylor , 1987]. The data are $0.55,0.93,1.25,1.36,1.49,1.52,1.58,1.61,1.64,1.68,1.73,1.81,2.00,0.74$, $1.04,1.27,1.39,1.49,1.53,1.59,1.61,1.66,1.68,1.76,1.82,2.01,0.77,1.11,1.28,1.42,1.50,1.54,1.60,1.62,1.66$, $1.69,1.76,1.84,2.24,0.81,1.13,1.29,1.48,1.50,1.55,1.61,1.62,1.66,1.70,1.77,1.84,0.84,1.24,1.30,1.48,1.51$, $1.55,1.61,1.63,1.67,1.70,1.78,1.89$. 
Table 2. Parameter estimates and goodness-of-fit statistics for various models fitted for $1.5 \mathrm{~cm}$ glass fibres data set

\begin{tabular}{|c|c|c|c|c|c|c|c|c|c|c|c|}
\hline \multirow{3}{*}{$\frac{\text { Model }}{\text { MO-HL-W }}$} & \multicolumn{4}{|c|}{ Estimates } & \multicolumn{4}{|c|}{ Statistics } & \multirow[b]{2}{*}{$A^{*}$} & \multirow[b]{2}{*}{ K-S } & \multirow[b]{2}{*}{ p-value } \\
\hline & $\delta$ & $\lambda$ & $\omega$ & $-2 \log L$ & $A I C$ & $A I C C$ & $B I C$ & $W^{*}$ & & & \\
\hline & $\begin{array}{c}8.3217 \\
(10.3885)\end{array}$ & $\begin{array}{c}0.6948 \\
(0.5651)\end{array}$ & $\begin{array}{c}3.2018 \\
(0.9478)\end{array}$ & 24.1 & 30.1 & 30.5 & 36.5 & 0.1057 & 0.5912 & 0.0999 & 0.5547 \\
\hline MOEGE & $\begin{array}{c}\alpha \\
1.3296 \times 10^{-3} \\
(0.3306)\end{array}$ & $\begin{array}{c}\gamma \\
10.7200 \\
(0.1849) \\
\end{array}$ & $\begin{array}{c}\lambda \\
5.8366 \\
(0.2071) \\
\end{array}$ & 31.9 & 37.9 & 38.4 & 44.4 & 0.4410 & 2.4309 & 0.9995 & $<2.200 \times 10^{-16}$ \\
\hline $\mathrm{EFr}$ & $\begin{array}{c}\alpha \\
0.04621 \\
(0.0153)\end{array}$ & $\begin{array}{c}\delta \\
0.4993 \\
(0.0147)\end{array}$ & $\begin{array}{c}\lambda \\
20.1145 \\
(6.1482)\end{array}$ & 189.1 & 195.1 & 195.5 & 201.6 & 1.1986 & 6.3098 & 0.4279 & $1.9210 \times 10^{-10}$ \\
\hline MOEFr & $\begin{array}{c}54074 \\
\left(3.8277 \times 10^{-8}\right)\end{array}$ & $\begin{array}{c}0.3858 \\
\left(6.0532 \times 10^{-2}\right)\end{array}$ & $\begin{array}{c}7.9253 \\
(0.8731) \\
\end{array}$ & 45.6 & 51.6 & 51.9 & 58.0 & 19.2509 & 122.7666 & 0.9997 & $<2.200 \times 10^{-16}$ \\
\hline EW & $\begin{array}{c}\alpha \\
1.8741 \\
(0.5608) \\
\end{array}$ & $\begin{array}{c}\beta \\
1.3803 \\
(0.3216) \\
\end{array}$ & $\begin{array}{c}\delta \\
15.3624 \\
(9.0935) \\
\end{array}$ & $\begin{array}{c}- \\
64.9\end{array}$ & 70.9 & 71.3 & 77.3 & 0.7088 & 3.8788 & 0.2487 & 0.0008 \\
\hline IWMO & $\begin{array}{c}\alpha \\
52636 \\
(9.7035) \\
\end{array}$ & $\begin{array}{c}\lambda \\
7.9256 \\
(0.1041) \\
\end{array}$ & $\begin{array}{c}\theta \\
2.5828 \\
(54.9189) \\
\end{array}$ & 45.6 & 51.6 & 51.9 & 58.0 & 0.4974 & 2.7509 & 0.1536 & 0.1020 \\
\hline APW & $\begin{array}{c}\alpha \\
10.8558 \\
(12.7241)\end{array}$ & $\begin{array}{c}\beta \\
4.4836 \\
(0.7632)\end{array}$ & $\begin{array}{c}\theta \\
0.1948 \\
(0.1083)\end{array}$ & 26.9 & 32.9 & 33.4 & 39.4 & 0.1686 & 0.9272 & 0.1225 & 0.3009 \\
\hline
\end{tabular}

The estimated variance-covariance matrix for MO-HL-W model on $1.5 \mathrm{~cm}$ glass fibres data set is given by

$$
\left[\begin{array}{ccc}
107.9206 & 5.6728 & -9.0626 \\
5.6728 & 0.3193 & -0.5279 \\
-9.0626 & -0.5279 & 0.8983
\end{array}\right]
$$

and the 95\% confidence intervals for the model parameters are given by $\delta \in[8.3217 \pm 20.3614], \lambda \in[0.6948 \pm 1.1076]$ and $\omega \in[3.2018 \pm 1.8577]$. Based on the results shown in Table 2 and the plots shown in Figures 4 (a) and 4 (b), we can conclude that the new proposed model performs better than the selected non-nested models on strength of $1.5 \mathrm{~cm}$ glass fibres.
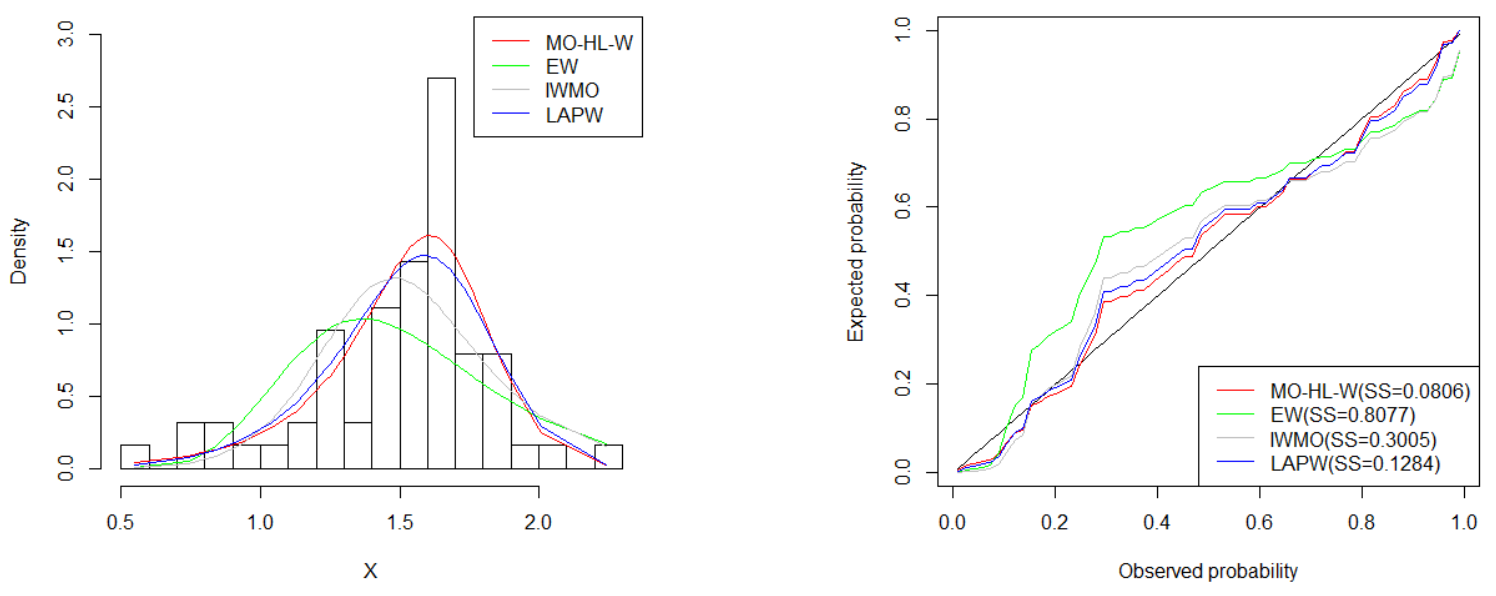

Figure 4. Fitted densities and probability plots for glass fibres data

\subsection{Silicon Nitride Data}

The second data set is on fracture toughness of silicon nitride measured in MPa $m^{1 / 2}$. The data set was also analyzed by [Nadarajah and Kotz , 2007] and also by [Ali et al. , 2015]. The data are 5.50, 5.00, 4.90, 6.40, 5.10, 5.20, 5.20, 5.00, $4.70,4.00,4.50,4.20,4.10,4.56,5.01,4.70,3.13,3.12,2.68,2.77,2.70,2.36,4.38,5.73,4.35,6.81,1.91,2.66,2.61$, $1.68,2.04,2.08,2.13,3.80,3.73,3.71,3.28,3.90,4.00,3.80,4.10,3.90,4.05,4.00,3.95,4.00,4.50,4.50,4.20,4.55$, $4.65,4.10,4.25,4.30,4.50,4.70,5.15,4.30,4.50,4.90,5.00,5.35,5.15,5.25,5.80,5.85,5.90,5.75,6.25,6.05,5.90$, 
$3.60,4.10,4.50,5.30,4.85,5.30,5.45,5.10,5.30,5.20,5.30,5.25,4.75,4.50,4.20,4.00,4.15,4.25,4.30,3.75,3.95$, $3.51,4.13,5.40,5.00,2.10,4.60,3.20,2.50,4.10,3.50,3.20,3.30,4.60,4.30,4.30,4.50,5.50,4.60,4.90,4.30,3.00$, 3.40, 3.70, 4.40, 4.90, 4.90, 5.00.

The estimated variance-covariance matrix for MO-HL-W model on silicon nitride data set is given by

$$
\left[\begin{array}{ccc}
21.4266 & 0.1708 & -6.2857 \\
0.1708 & 0.0013 & -0.0516 \\
-6.2857 & -0.0516 & 1.9136
\end{array}\right]
$$

and the 95\% confidence intervals for the model parameters are given by $\delta \in[2.8087 \pm 9.0726], \lambda \in[0.0134 \pm 0.0732]$ and $\omega \in[3.3255 \pm 2.7114]$. Results in Table 3 show that the MO-HL-W distribution performs better than the various non-nested models on silicon nitride data. We can also deduce from the fitted densities and probability plots that the MO-HL-W distribution fits the silicon nitride data better than the selected non-nested models.

Table 3. Parameter estimates and goodness-of-fit statistics for various models fitted for silicon nitride data set

\begin{tabular}{|c|c|c|c|c|c|c|c|c|c|c|c|}
\hline \multirow[b]{2}{*}{ Model } & \multicolumn{4}{|c|}{ Estimates } & \multicolumn{4}{|c|}{ Statistics } & \multirow[b]{2}{*}{$A^{*}$} & \multirow[b]{2}{*}{$\mathrm{K}-\mathrm{S}$} & \multirow[b]{2}{*}{ p-value } \\
\hline & $\delta$ & $\lambda$ & $\omega$ & $-2 \log L$ & $A I C$ & $A I C C$ & $B I C$ & $W^{*}$ & & & \\
\hline MO-HL-W & $\begin{array}{c}2.8087 \\
(4.6289)\end{array}$ & $\begin{array}{c}0.0134 \\
(0.0374)\end{array}$ & $\begin{array}{c}3.3255 \\
(1.3834)\end{array}$ & 335.6 & 341.6 & 341.8 & 349.9 & 0.0506 & 0.3047 & 0.0523 & 0.9009 \\
\hline MOEGE & $\begin{array}{c}\alpha \\
1.0718 \times 10^{-2} \\
\left(7.0715 \times 10^{-3}\right)\end{array}$ & $\begin{array}{c}\gamma \\
20.7600 \\
\left(3.5685 \times 10^{-5}\right)\end{array}$ & $\begin{array}{c}\lambda \\
1.7321 \\
(0.1434)\end{array}$ & 340.3 & 346.3 & 346.5 & 354.6 & 0.2326 & 1.4637 & 0.9926 & $<2.2000 \times 10^{-16}$ \\
\hline EFr & $\begin{array}{c}\alpha \\
0.0555 \\
(0.0244)\end{array}$ & $\begin{array}{c}\delta \\
1.5719 \\
(0.0359)\end{array}$ & $\begin{array}{c}\lambda \\
18.4091 \\
(7.7522)\end{array}$ & 586.9 & 592.9 & 593.2 & 601.3 & 1.3837 & 7.6851 & 0.3861 & $7.772 \times 10^{-16}$ \\
\hline MOEFr & $\begin{array}{c}2407.7 \\
\left(7.7867 \times 10^{-6}\right)\end{array}$ & $\begin{array}{c}1.4344 \\
(0.1296)\end{array}$ & $\begin{array}{c}7.0579 \\
(0.5495)\end{array}$ & 356.6 & 362.6 & 362.9 & 370.9 & 38.2495 & 235.4782 & 0.9989 & $<2.2000 \times 10^{-16}$ \\
\hline EW & $\begin{array}{c}\alpha \\
0.7015 \\
(0.2973)\end{array}$ & $\begin{array}{c}\beta \\
1.1441 \\
(0.2012)\end{array}$ & $\begin{array}{c}\delta \\
23.9388 \\
(12.5289) \\
\end{array}$ & $\begin{array}{c}- \\
381.5 \\
-\end{array}$ & 387.5 & 387.7 & 395.8 & 0.6594 & 3.8924 & 0.1676 & 0.0025 \\
\hline IWMO & $\begin{array}{c}\alpha \\
2407.7 \\
\left(8.9422 \times 10^{-7}\right)\end{array}$ & $\begin{array}{c}\lambda \\
7.0579 \\
(0.5495)\end{array}$ & $\begin{array}{c}\theta \\
0.6972 \\
(0.0630)\end{array}$ & 356.6 & 362.6 & 362.9 & 370.9 & 0.3596 & 2.2543 & 0.0804 & 0.4241 \\
\hline APW & $\begin{array}{c}\alpha \\
7.4246 \\
(0.0201)\end{array}$ & $\begin{array}{c}\beta \\
3.8975 \\
(0.2965)\end{array}$ & $\begin{array}{c}\theta \\
0.0039 \\
(0.0019)\end{array}$ & 335.8 & 341.8 & 342.0 & 350.2 & 0.0571 & 0.3591 & 0.0560 & 0.8492 \\
\hline
\end{tabular}
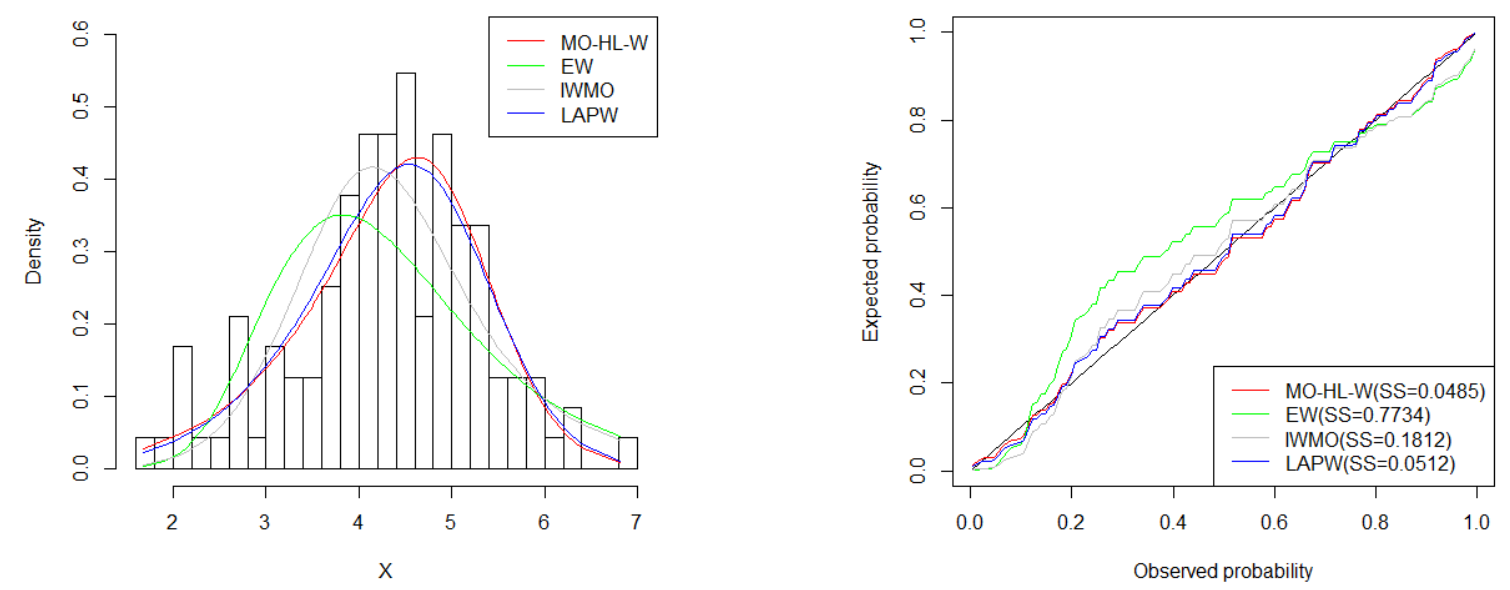

Figure 5. Fitted densities and probability plots for silicon nitride data 


\subsection{Turbocharger Failure Times Data}

The third data set represents failure times $\left(10^{3} h\right)$ of turbocharger of one type of engine as report by [Xu et al. , 2003]. The data are 1.6, 2.0, 2.6, 3.0, 3.5, 3.9, 4.5, 4.6, 4.8, 5.0, 5.1, 5.3, 5.4, 5.6, 5.8, 6.0, 6.0, 6.1, 6.3, 6.5, 6.5, 6.7, 7.0, 7.1, 7.3, 7.3, $7.3,7.7,7.7,7.8,7.9,8.0,8.1,8.3,8.4,8.4,8.5,8.7,8.8,9.0$.

Table 4. Parameters estimates and goodness-of-fit statistics for various models fitted for turbocharger failure times data set

\begin{tabular}{|c|c|c|c|c|c|c|c|c|c|c|c|}
\hline \multirow[b]{2}{*}{ Model } & \multicolumn{4}{|c|}{ Estimates } & \multicolumn{4}{|c|}{ Statistics } & \multirow[b]{2}{*}{$A^{*}$} & \multirow[b]{2}{*}{$\mathrm{K}-\mathrm{S}$} & \multirow[b]{2}{*}{ p-value } \\
\hline & $\delta$ & $\lambda$ & $\omega$ & $-2 \log L$ & $A I C$ & $A I C C$ & $B I C$ & $W^{*}$ & & & \\
\hline MO-HL-W & $\begin{array}{c}2.4294 \\
(2.8327) \\
\end{array}$ & $\begin{array}{c}0.0096 \\
(0.0203)\end{array}$ & $\begin{array}{c}2.7877 \\
(0.8728)\end{array}$ & 162.6 & 168.6 & 169.3 & 173.7 & 0.0496 & 0.3766 & 0.0918 & 0.8889 \\
\hline MOEGE & $\begin{array}{c}\alpha \\
0.116 \\
(0.0280) \\
\end{array}$ & $\begin{array}{c}\gamma \\
3.1108 \\
(0.6155) \\
\end{array}$ & $\begin{array}{c}\lambda \\
0.8755 \\
(0.1246) \\
\end{array}$ & 167.1 & 173.1 & 173.8 & 178.2 & 0.1183 & 0.8382 & 0.9932 & $<2.2000 \times 10^{-16}$ \\
\hline $\mathrm{EF}$ & $\begin{array}{c}\alpha \\
0.0398 \\
(0.0065)\end{array}$ & $\begin{array}{c}\delta \\
1.4314 \\
(0.0414)\end{array}$ & $\begin{array}{c}\lambda \\
18.2212 \\
(0.6291)\end{array}$ & 253.7 & 259.7 & 260.3 & 264.7 & 0.6684 & 3.7808 & 0.4145 & $2.1430 \times 10^{-6}$ \\
\hline MOEF & $\begin{array}{c}4836.2 \\
\left(1.1593 \times 10^{-5}\right)\end{array}$ & $\begin{array}{c}1.0782 \\
(0.2678)\end{array}$ & $\begin{array}{c}4.8391 \\
(0.6512)\end{array}$ & 177.4 & 183.4 & 184.1 & 188.5 & 12.8752 & 78.7089 & 0.9987 & $<2.2000 \times 10^{-16}$ \\
\hline EW & $\begin{array}{c}\alpha \\
0.7549 \\
(0.6721) \\
\end{array}$ & $\begin{array}{c}\beta \\
0.8389 \\
(0.0400) \\
\end{array}$ & $\begin{array}{c}\delta \\
17.9156 \\
(22.6988) \\
\end{array}$ & $\begin{array}{c}- \\
187.4 \\
- \\
\end{array}$ & 193.4 & 194.1 & 198.5 & 0.3195 & 1.9997 & 0.1672 & 0.2134 \\
\hline IWMO & $\begin{array}{c}\alpha \\
4836.3 \\
\left(8.4139 \times 10^{-6}\right)\end{array}$ & $\begin{array}{c}\lambda \\
4.8391 \\
(0.6512)\end{array}$ & $\begin{array}{c}\theta \\
0.9274 \\
(0.2303)\end{array}$ & 177.4 & 183.4 & 18401 & 188.5 & 0.2153 & 1.4129 & 0.1438 & 0.3796 \\
\hline LATW & $\begin{array}{c}\alpha \\
4.3051 \\
(0.0444)\end{array}$ & $\begin{array}{c}\beta \\
3.3068 \\
(0.4576) \\
\end{array}$ & $\begin{array}{c}\theta \\
0.0024 \\
(0.0022)\end{array}$ & 163.8 & 169.8 & 170.5 & 174.9 & 0.0621 & 0.4691 & 0.0986 & 0.8320 \\
\hline
\end{tabular}

The estimated variance-covariance matrix for MO-HL-W model on turbocharger failure times data set is given by

$$
\left[\begin{array}{ccc}
8.0241 & 0.0528 & -2.1838 \\
0.0528 & 0.0004 & -0.0176 \\
-2.1838 & -0.0176 & 0.7617
\end{array}\right]
$$

and the $95 \%$ confidence intervals for the model parameters are given by $\delta \in[2.4294 \pm 5.5521], \lambda \in[0.0096 \pm 0.0399]$ and $\omega \in[2.7877 \pm 1.7106]$. Results in Table 4 and plots in Figures 6 (a) and 6 (b) show that the MO-HL-W distribution performs better than the various non-nested models on failure times of turbocharger data.
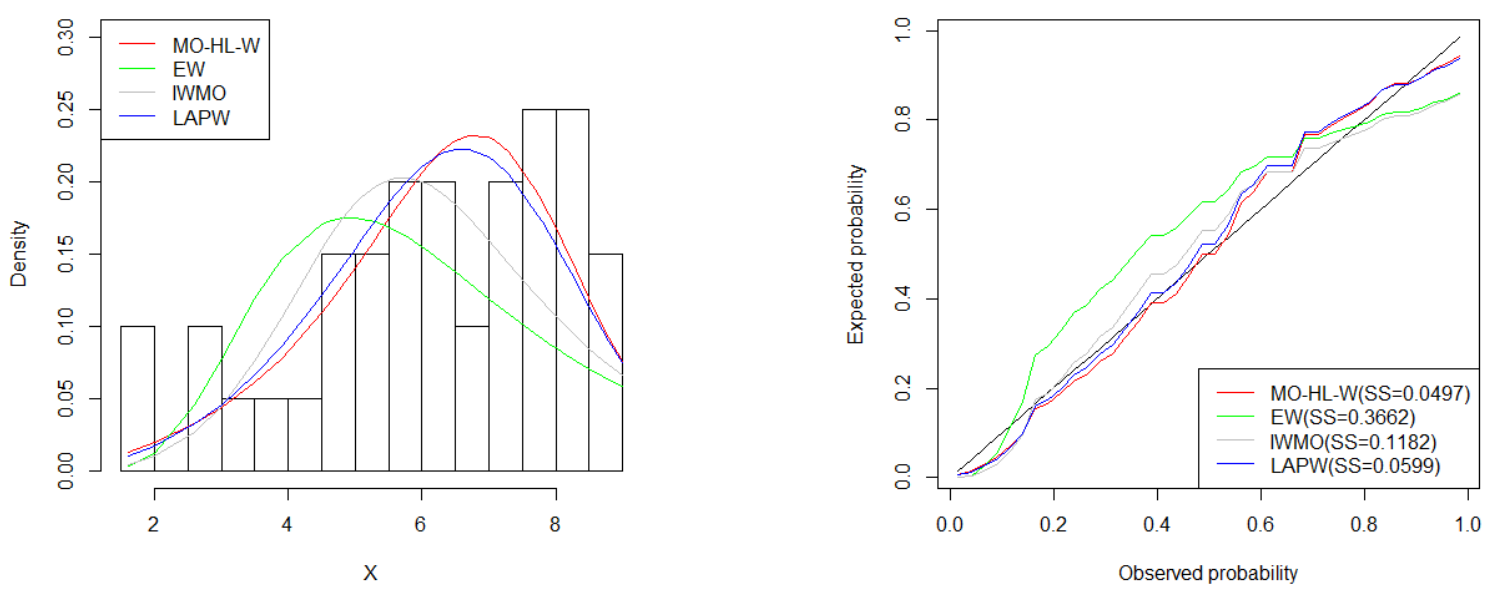

Figure 6. Fitted densities and probability plots for turbocharger failure times data 


\section{Concluding Remarks}

A new model was developed, which is referred to as the Marshall-Olkin-Half Logistic-G family of distributions. The new distribution is a linear combination of the Exp-G distribution. The new proposed distribution can be applied to heavily skewed data and also data that have non-monotonic hazard rate shapes. From real data examples presented in Tables 2, 3 and 4, we conclude that the MO-HL-W distribution performs better than several competing non-nested models.

\section{Acknowledgement}

The authors are particularly grateful to the anonymous referees and Editor for their valuable comments and suggestions that led to this improved version.

\section{References}

Afify, A. Z., Altun, E., Alizadeh, M., Ozel, G., \& Hamedani, G. G. (2017). The odd exponentiated half-logistic-G family: properties, characterizations and applications. Chilean Journal of Statistics, 8(2), 65-91.

Ali, A., Hasnain, S. A., \& Ahmad, M. (2015). Modified Burr XII distribution, properties and applications, Pakistan Journal of Statistics, 31(6), 697-708.

Alexander, C., Cordeiro, G. M., Ortega, E. M. M., \& Sarabia, J. M. (2012). Generalized beta generated distributions. Computational Statistics and Data Analysis, 56, 1880-1897. https://doi.org/10.1016/j.csda.2011.11.015

Alizadeh, A, Cordeiro, G. M., Brito, E., \& Demétrio, C. G. (2015a). The beta Marshall-Olkin family of distributions. Journal of Statistical Distributions and Applications, 4(2), 1-18. https://doi.org/10.1186/s40488-015-0027-7

Alizadeh, A., Emadi, M., Doostparast, M., Cordeiro, G. M., Ortega, E., \& Pescim, R. (2015b). A new family of distributions: The Kumaraswamy odd log-logistic, properties and applications. Hacettepe Journal of Mathematics and Statistics, 44, 1491-1512.

Alzaatreh, A., Famoye, F., \& Lee, C. (2013). A new method for generating families of continuous distributions. Metron, 71, 63-79. https://doi.org/10.1007/s40300-013-0007-y

Barreto-Souza, W., \& Bakouch., H. S. (2013). A new lifetime model with decreasing failure rate, Statistics: A Journal of Theoretical and Applied Statistics, 47, 465-476. https://doi.org/10.1080/02331888.2011.595489

Barreto-Souza, W., Lemonte, A. J., \& Cordeiro, G. M. (2013). General results for the Marshall and Olkin's family of distributions. Annals of the Brazilian Academy of Sciences, 85(1), 3-21. https://doi.org/10.1590/S0001-37652013000100002

Bourguignon, M., Silva, R. B., \& Cordeiro, G. M. (2014). The Weibull-G family of probability distributions. Journal of Data Science, 12, 53-68.

Chakraborty, S., \& Handique, L. (2017). The generalized Marshall-Olkin-Kumaraswamy-G family of distributions, Journal of Data Science, 15(3), 391-422.

Chambers, J., Cleveland, W., Kleiner, B., \& Tukey, P. (1983). Graphical methods of data analysis, Chapman and Hall.

Chen, G., \& Balakrishnan, N. (1995). A general purpose approximate goodness-of-fit test. Journal of Quality Technology, 27(2), 154-161. https://doi.org/10.1080/00224065.1995.11979578

Cordeiro, G. M., \& de Castro, M. (2011). A new family of generalized distributions. Journal of Statistical Computation and Simulation, 81, 883-898. https://doi.org/10.1080/00949650903530745

Cordeiro, G. M., Ortega, E. M., \& da Cunha, D. C. (2013). The exponentiated generalized class of distributions. Journal of Data Science, 11, 1-27.

Cordeiro, G. M., Alizadeh, M., \& Diniz Marinho, P. R. (2016). The type I half-logistic family of distributions. Journal of Statistical Computation and Simulation, 86(4), 707-728.

Cordeiro, G. M., Alizadeh, M., \& Ortega, E. M. M. (2014). The exponentiated half logistic family of distributions: properties and applications. Journal of Probability and Statistics, 81, 1-21. https://doi.org/10.1155/2014/864396

Cordeiro, G. M., Alizadeh, M., Tahir, M. H., Mansoor, M., Bourguignon, M., \& Hamedani, G. G. (2015). The beta odd log-logistic family of distributions. Hacettepe Journal of Mathematics and Statistics, forthcoming. 
Cordeiro, G. M., Alizadeh, M., Ozel, G., Hosseini, B., Ortega, E. M. M., \& Altun, E. (2017). The generalized odd loglogistic family of distributions: properties, regression models and applications. Journal of Statistical Computation and Simulation, 87, 908-932.

Eugene, N., Lee, C., \& Famoye, F. (2002). Beta-normal distribution and its applications. Communications in Statistics Theory and Methods, 31, 497-512. https://doi.org/10.1081/STA-120003130

Ghitany, M. E., AL-Hussaini, E. K., \& AL-Jarallah. (2005). Marshall-Olkin Extended Weibull distribution and its application to Censored data. Journal of Applied Statistics, 32(10), 1025-1034. https://doi.org/10.1080/02664760500165008

Gleaton, J. U., \& Lynch, J. D. (2004). On the distribution of the breaking strain of a bundle of brittle elastic fibers. Advances in Applied Probability, 36, 98-115. https://doi.org/10.1239/aap/1077134466

Kumar, D. (2016). Ratio and inverse moments of Marshall-Olkin extended Burr type III distribution based on lower generalized order statistics. Journal of Data Science, 14(1), 53-66.

Lazhar, B. (2017). Marshall-Olkin extended generalized Gompertz distribution, Journal of Data Science, 15(2), 239-266.

Lepetu, L., Oluyede, B. O, Makubate, B., Foya, S., \& Mdlongwa, P. (2017). Marshall-Olkin log-logistic extended Weibull distribution: theory, properties and applications. Journal of Data Science, 15, 691-722.

Marshall, A. W., \& Olkin, I. (1997). A new method for adding a parameter to a family of distributions with application to the exponential and Weibull families. Biometrika, 84(3), 641-652.

Nadarajah, S., \& Kotz, S. (2007). On the alternative to Weibull function, Engineering Fracture Mechanics, 74(3), 451-456. https://doi.org/10.1016/j.engfracmech.2006.06.007

Nadarajah S., \& Kotz, S. (2003). The exponentiated Fréchet distribution, interstat.statjournals.net/YEAR/2003/articles/0312001.pdf.

Nassar, M., Alzaatreh, A., Mead, M., \& Abo-Kasem, O. (2018). Alpha power Weibull distribution: properties and applications, Communications in Statistics - Theory and Methods, 46(20), 10236-10252. https://doi.org/10.1080/03610926.2016.1231816

Nofal, Z. M., Afify, A. Z., Yousof, H. M., \& Cordeiro, G. M. (2017). The generalized transmuted-G family of distributions. Communications in Statistics - Theory and Methods, 46, 4119-4136.

Pakungwati, R. M., Widyaningsih, Y., \& Lestari, D. (2018). Marshall-Olkin extended inverse Weibull distribution and its application. Journal of Physics. https://doi.org/10.1088/1742-6596/1108/1/012114

Pal, M., Ali, M. M., \& Woo, J. (2006). Exponentiated Weibull distribution. STATISTICA, anno LXVI(2), 139-147.

Rényi, A. (1961). On measures of entropy and information. Proceedings of the Fourth Berkeley Symposium on Mathematical Statistics and Probability. The Regents of the University of California,Volume 1.

Santos-Neo, M., Bourguignon, M., Zea, L. M., \& Nascimento, A. D. C. (2014). The Marshall-Olkin extended Weibull Family of distributions, Journal of Statistical Distributions and Applications, 1-9.

Shannon, C. E. Predection and entropy of printed english (1951). The Bell System Technical Journal, 30(1), 50-64. https://doi.org/10.1002/j.1538-7305.1951.tb01366.x

Shaw, W. T., \& Buckley, I. R. C. (2009). The alchemy of probability distributions: beyond Gram-Charlier expansions and a skew-kurtotic-normal distribution from a rank transmutation map. arXiv: 0901.0434

Smith, R. L., \& Naylor, J. C. (1987). A Comparison of maximum likelihood and Bayesian estimators for the threeparameter Weibull distribution. Applied Statistics, 36, 358-369. https://doi.org/10.2307/2347795

Torabi, H., \& Montazeri, N. H. (2014). The logistic-uniform distribution and its application. Communications in Statistics - Simulation and Computation, 43, 2551-2569. https://doi.org/10.1080/03610918.2012.737491

Xu, K., Xie, M., Tang, L. C., \& Ho, S. L. (2003). Application of neural networks in forecasting engine systems reliability. Applied Software Computing, 2(4), 255-268. https://doi.org/10.1001/jama.1986.03370020118051

Zhang, T., \& Xie, M. (2007). Failure data analysis with extended Weibull distribution, Commun. Stat. Simul., 36, 579-592. https://doi.org/10.1080/03610910701236081 
Zografos, K., \& Balakrishnan, N. (2009). On families of beta and generalized gamma generated distributions and associated inference. Statistical Methodology, 6, 344-362. https://doi.org/10.1016/j.stamet.2008.12.003

\section{Copyrights}

Copyright for this article is retained by the author(s), with first publication rights granted to the journal.

This is an open-access article distributed under the terms and conditions of the Creative Commons Attribution license (http://creativecommons.org/licenses/by/4.0/). 